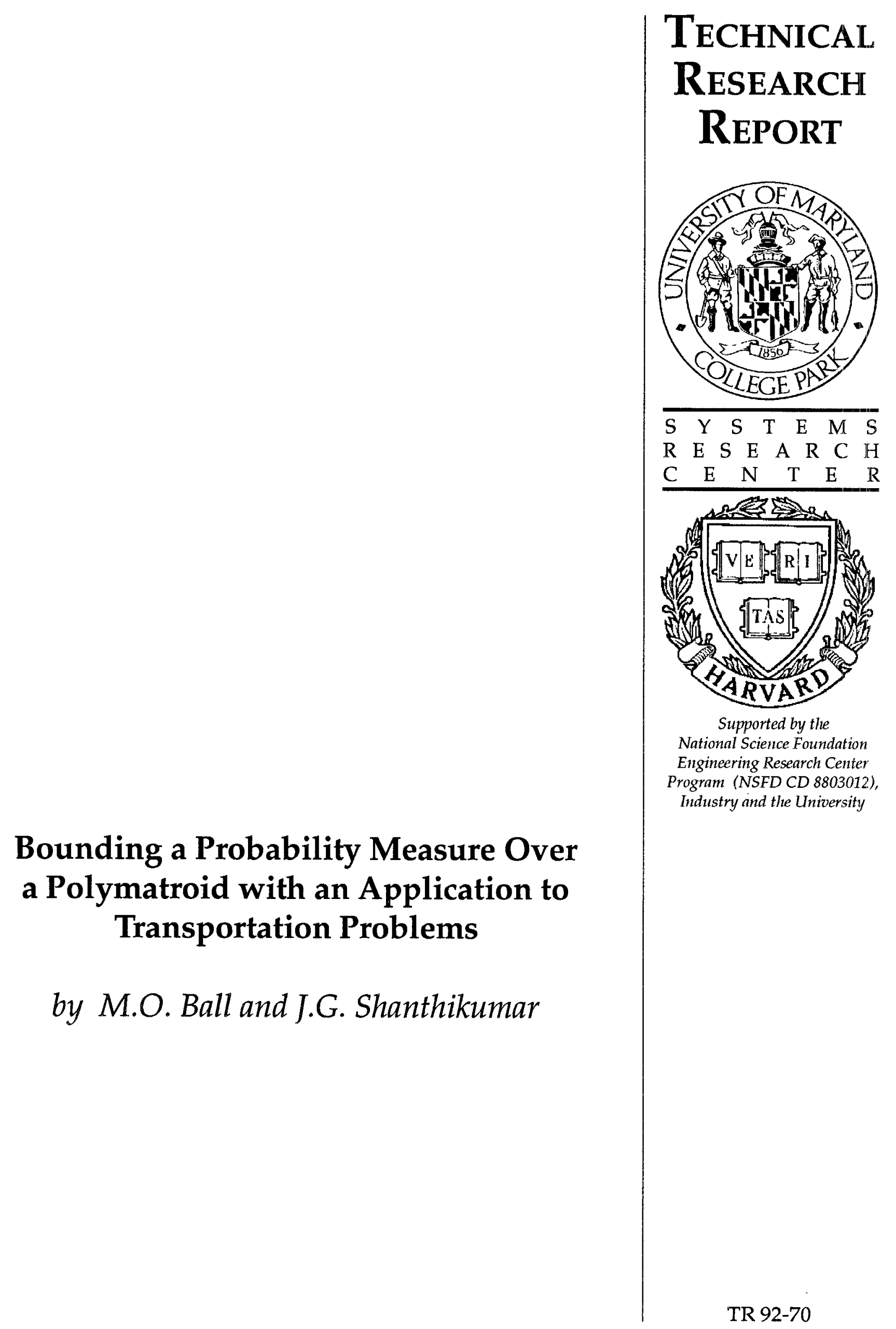





\title{
BOUNDING A PROBABILITY MEASURE OVER A POLYMATROID WITH AN APPLICATION TO TRANSPORTATION PROBLEMS
}

\author{
Michael O. Ball \\ College of Business and Management \\ and \\ Systems Research Center \\ University of Maryland at College Park \\ College Park, MD 20742 \\ and \\ J. George Shanthikumar \\ Walter A. Haas School of Business \\ University of California at Berkeley \\ Berkeley, CA 94720
}

April 1992

Michael O. Ball was supported in part by NSF grant CDR-8803012 and J. George Shanthikumar was supported in part by NSF Grant DDM-9113008. 



\begin{abstract}
For $M=\{1, \ldots, m\}$ and a supermodular set function $f: 2^{M} \rightarrow \mathcal{R}$, define the polymatroid $\mathcal{P}(M, f)=\left\{\mathbf{x}: \sum_{j \in A} x_{j} \geq f(A), A \subseteq M ; \mathbf{x} \in \mathcal{R}^{m}\right\}$. We develop a bound for the probability $P\{\mathbf{X} \in \mathcal{P}(M, f)\}$, where $\mathbf{X}$ is a random $m$-vector. In particular we show that if $X, X_{1}, \ldots, X_{m}$, are independent and identically distributed non-negative random variables with new better than used (NBU) distribution function, then $P\{\mathrm{X} \in \mathcal{P}(M, f)\} \geq$ $P\{X \geq f(\hat{M})\}$, where $\hat{M}=\arg \max \{f(A): A \subseteq M\}$. We apply this result to transportation problems with random supply and/or demand. Suppose we have a set $K=\{1, \ldots, k\}$ of $k$ supply nodes with a random supply $S_{i}, i \in K$ and a set $N=\{1, \ldots, n\}$ of $n$ demand nodes with a random demand $D_{j}, j \in N$. Let $\beta$ be the probability that the random demand can be met by the random supply. If the demand and supply are mutually independent, $D_{1}, \ldots, D_{n}$, are independent, and $S_{1}, \ldots, S_{k}$, are independent and have NBU distribution function, then $\beta \leq \Pi_{j=1}^{n} P\left\{Z_{j} \geq D_{j}\right\}$, where $Z_{j}=\sum_{i \in K} S_{i} r_{i j}, j \in N$ and $r_{i j}$ takes the value one if node $i$ can supply the demand node $j$ and zero otherwise ( $i \in K$ and $j \in N)$.
\end{abstract}

Key Words: Chance constraint; polymatroid; bounds; transportation problem; NBU distribution; supermodularity. 



\section{Introduction}

Often in the design of stochastic systems one encounters a mathematical programming formulation that has either a constraint or an objective function that relates to the probability that a collection of random variables $\mathbf{X}=\left(X_{1}, \ldots, X_{m}\right)$ associated with the stochastic system fall within a constraint set, say $\mathcal{V}$. That is, we are concerned with the probability $P\{\mathrm{X} \in \mathcal{V}\}$. When either the constraint set and/or the parameters of the random variables are themselves decision variables, it is desirable that one has a simple (explicit) formula for $P\{\mathbf{X} \in \mathcal{V}\}$. If such a simple formula does not exist, then it is desirable to have a tight upper and/or lower bound that has a simple formula. Suppose $\mathcal{V}$ is an upper [lower] set (i.e., for any $\mathbf{x} \in \mathcal{V}, \mathbf{x} \leq[\geq] \mathbf{y} \rightarrow \mathbf{y} \in \mathcal{V}$ ). Then for any feasible $\mathbf{x}$ (i.e., $\mathbf{x} \in \mathcal{V})$, we have

$$
P\{\mathbf{X} \in \mathcal{V}\} \geq P\{\mathbf{X} \geq \mathbf{x}\} \quad[P\{\mathbf{X} \in \mathcal{V}\} \geq P\{\mathbf{X} \leq \mathbf{x}\}]
$$

When $X_{1}, \ldots, X_{m}$, are independent random variables, one has

$$
P\{\mathbf{X} \in \mathcal{V}\} \geq \Pi_{i=1}^{m} P\left\{X_{i} \geq x_{i}\right\} \quad\left[P\{\mathbf{X} \in \mathcal{V}\} \geq \Pi_{i=1}^{m} P\left\{X_{i} \leq x_{i}\right\}\right]
$$

Therefore if we can find a feasible $\mathbf{x}$ (preferably one that maximizes the right hand side of (1)) we can use the above bounds. If only the parameters of the random variables are decision variables and the constraint set $\mathcal{V}$ is fixed then we can find a feasible $\mathbf{x}$ and fix it in the bound. On the other hand if the constraint set $\mathcal{V}$ itself is a function of the decision variables, then a feasible $\mathbf{x}$ that is a simple function of the decision variables must be chosen. In this paper we consider such a case in which the constraint set is a Polymatroid where the rank function is a function of the decision variables.

In Section 2 we develop a bound for the probability $P\{\mathbf{X} \in \mathcal{V}\}$, where $\mathcal{V}$ is a Polymatroid. Our interest in this bound is based on its applicability to a certain stochastic transportation problem. Suppose we have a set $K=\{1, \ldots, k\}$ of $k$ supply nodes and a set $N=\{1, \ldots, n\}$ of $n$ demand nodes. Let $r_{i j}$ take the value one if node $i$ can supply the demand node $j$ and zero otherwise $(i \in K$ and $j \in N)$. There is a random demand 
DEMANO NODES •
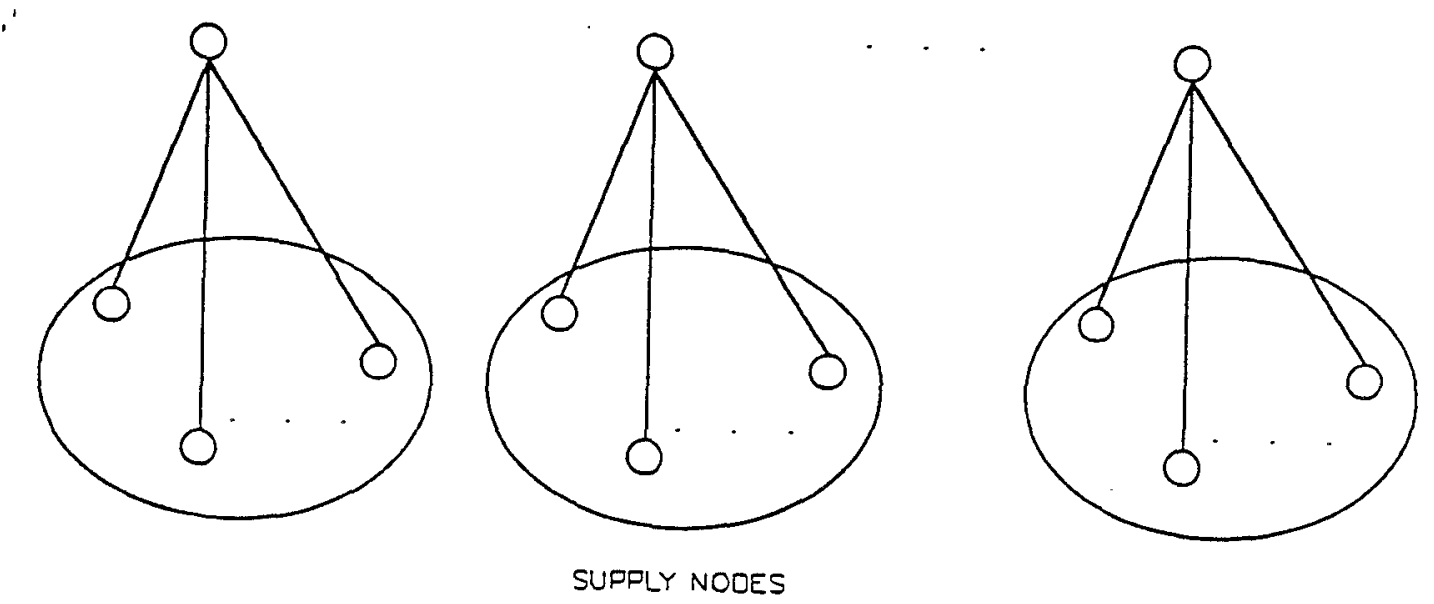

Figure 1A: Independent Supply Sources

of $D_{j}$ units at node $j, j \in N$ and a random supply of $S_{i}$ units at node $i, i \in K$. Let $\beta$ be the probability that the random demand can be met by the random supply. Now as is illustrated in Figure 1A, suppose that each demand node has independent supply sources so that for each supply node $i$ there is exactly one $j$ with $r_{i j}=1$. Then, if the demands and supplies are mutually independent, $D_{1}, \ldots, D_{n}$, are independent, and $S_{1}, \ldots, S_{k}$, are independent, it follows that $\beta=\prod_{j=1}^{n} P\left\{Z_{j} \geq D_{j}\right\}$, where $Z_{j}=\sum_{i \in K} S_{i} r_{i j}, j \in N$. In section 3, we use the bound of $P\{\mathbf{X} \in \mathcal{V}\}$, developed in Section 2 to derive a bound on $\beta$ for the case illustrated in Figure 1B where the demand nodes do not have indépendent supply: sources, i.e. demand nodes have overlapping sources. Specifically, we show (Corollary 1) that if, $D_{1}, \ldots, D_{n}$, are independent, and $S_{1}, \ldots, S_{k}$, are independent and have new better than used distribution functions, then $\beta \leq \Pi_{j=1}^{n} P\left\{Z_{j} \geq D_{j}\right\}$. That is, when one treats the supply sources as if they were independent the resulting calculation produces an upper bound on the actual probability when the supplies have new better than used distribution functions. 
DEMANO NODES

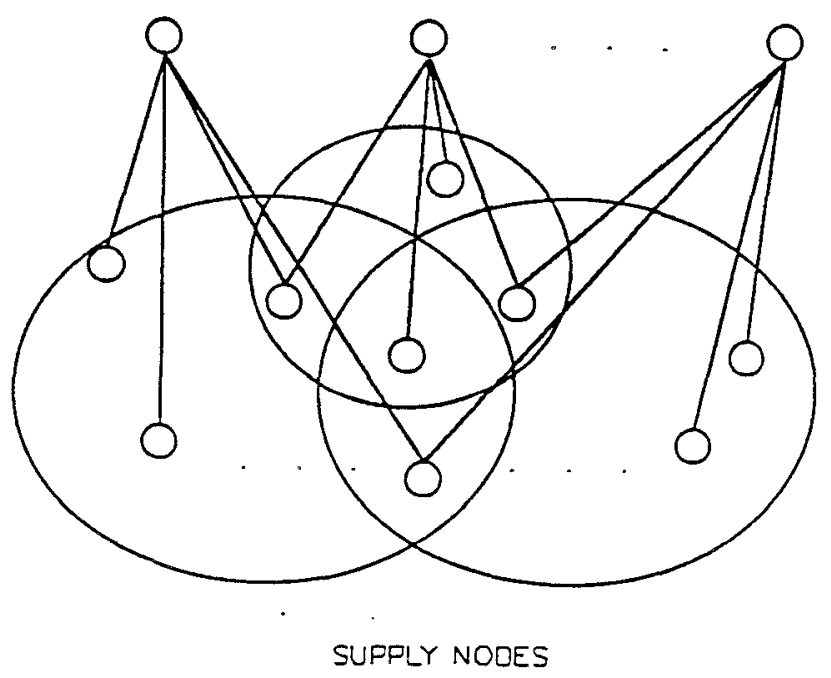

Figure 1B: Overlapping Supply Sources

In Ball and Lin 1992, this bound is used within an integer programming model for emergency services vehicle location. Constraints for the location model are generated from certain stochastic transportation problems illustrated in Figure 2.. The demand nodes, which have deterministic demand levels, correspond to locations for emergency service vehicles that can service a particular (new) call within an acceptable time limit. The supply nodes correspond to geographic locations that can generate other calls for the emergency service vehicles. If all the vehicles that can service the new call are busy then there must be a feasible 'solution to the transportation problem. Thus; the probability that there is a feasible solution to the transportation problem is an upper bound on the probability that the new call does not receive immediate service. The upper bound on this probability is embedded into an integer programming model that chooses emergency service vehicle locations and determines the number of vehicles to place at each location. This upper bound serves as the basis for several constraints since calls arising from locations scattered throughout a geographic region are modeled simultaneously. The model produces 
a solution that guarantees that the upper bound is less than or equal to a specified limit. This in turn implies that the probability itself will respect this limitation. The product form of the upper bound enables the construction of linear constraints using logarithms.

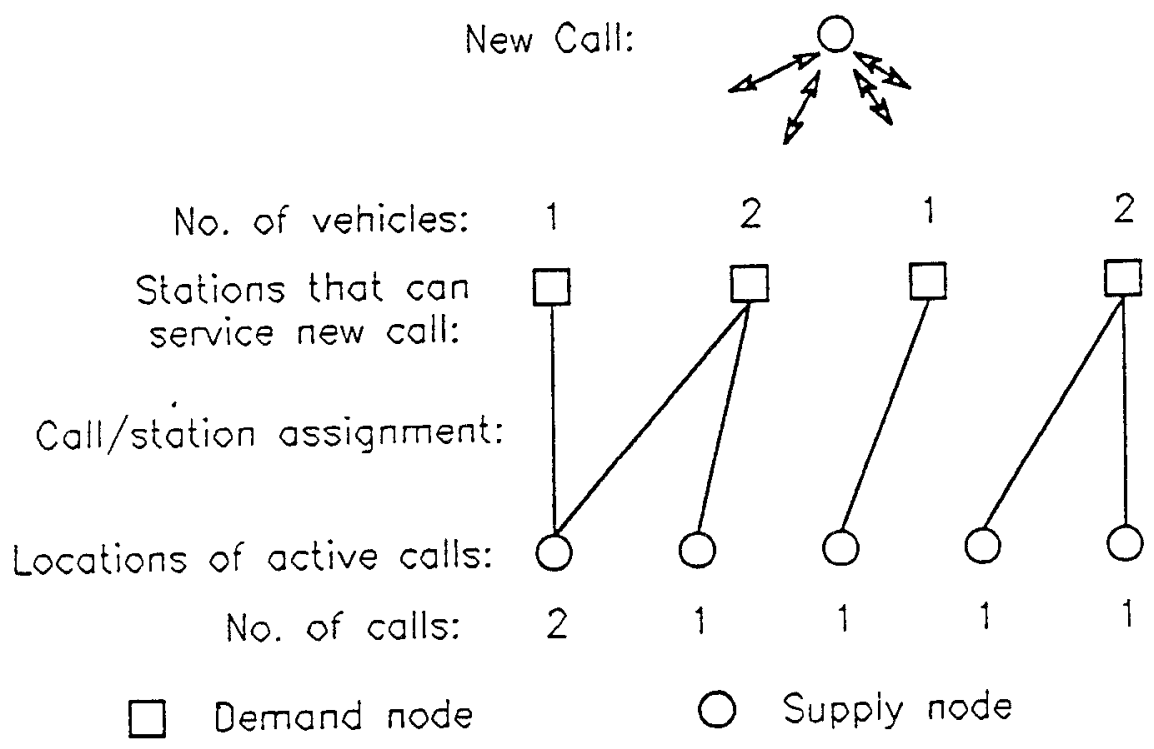

Figure 2: Emergency Services Transportation Problem

\section{A Probability Measure Over a Polymatroid}

For $M=\{1, \ldots, m\}$ let $2^{M}=\{A: A \subseteq M\}$ be the power set of $M$ and $f: 2^{M} \rightarrow \mathcal{R}$ with $f(\emptyset) \leq 0$ be a supermodular set function (i.e., for any $A, B \in 2^{M}$ we have $f(A \cup B)+$ $f(A \cap B) \geq f(A)+f(B))$. Now define the polymatroid $\mathcal{P}(M, f)$ by

$$
\mathcal{P}(M, f)=\left\{\mathrm{x}: \sum_{j \in A} x_{j} \geq f(A), A \in 2^{M} ; \mathrm{x} \in \mathcal{R}^{m}\right\} .
$$

Note that we require neither the monotonicity of $f$ nor the non-negativity of $f$ and $\mathbf{x}$ in this definition. We are interested in the probability $P\{\mathbf{X} \in \mathcal{P}(M, f)\}$ for some non-negative random vector $\mathrm{X}=\left(X_{1}, \ldots, X_{m}\right)$. Since $\mathcal{P}(M, f)$ is an upperset, for any $\mathrm{x} \in \mathcal{P}(M, f)$ (i.e., feasible $\mathbf{x}$ )

$$
P\{\mathrm{X} \in \mathcal{P}(M, f)\} \geq P\{\mathrm{X} \geq \mathbf{x}\}
$$


Of course the best bound within this class is obtained by maximizing the right hand side of the above inequality over all $\mathbf{x} \in \mathcal{P}(M, f)$. For example, when $X_{1}, \ldots, X_{m}$ are independent and have logconcave survival functions, a marginal allocation algorithm can be applied to maximize the lower bound. But, in this section, we will only consider a feasible $\mathbf{x}$ which is easy to find. Such an $\mathbf{x}$ can be constructed using basic ideas from the theo'ry of polymatroids (e.g., see Nemhauser and Wolsey 1988, Chapter III.3). Specifically, let $\pi$ be any permutation of $\{1, \ldots, m\}$ and define $S_{k}=\{\pi(1), \ldots, \pi(k)\}, k=1, \ldots, m$. Set

$$
\begin{gathered}
x_{1}(\pi)=f\left(S_{1}\right) \\
x_{j}(\pi)=f\left(S_{j}\right)-f\left(S_{j-1}\right), j=2, \ldots, m .
\end{gathered}
$$

Then $\mathbf{x}(\pi)=\left(x_{1}(\pi), \ldots, x_{m}(\pi)\right)$ is a vertex of $\mathcal{P}(M, f)$ and therefore

$$
P\{\mathbf{X} \in \mathcal{P}(M, f)\} \geq P\{\mathbf{X} \geq \mathbf{x}(\pi)\}
$$

If $\mathrm{X}$ is a vector of independent random variables, then

$$
P\{\mathrm{X} \in \mathcal{P}(M, f)\} \geq \Pi_{j=1}^{m} P\left\{X_{j} \geq x_{j}(\pi)\right\}
$$

We now derive a more interesting bound by assuming special structure on the random variables. A non-negative random variable, $X$, has the new better than used (NBU) property if for any $a, b \geq 0$, we have $P\{X \geq a\} P\{X \geq b\} \geq P\{X \geq a+b\}$, e.g., see Ross 1983. Suppose $X, X_{1}, \ldots, X_{m}$ are non-negative, i.i.d. random variables with an NBU distribution function. Now since $f(M)=\sum_{j=1}^{m} x(\pi)$, it follows from the NBU property that if $x_{j}(\pi) \geq 0, j=1, \ldots, m$, then

$$
P\{\mathrm{X} \in \mathcal{P}(M, f)\} \geq P\{X \geq f(M)\}
$$

The non-negativity of $\mathbf{x}(\pi)$ will be guaranteed by the monotonicity or the non-negativity of $f$ (which we did not require). We will now derive a bound similar to the above even when $f$ is not monotone. Specifically we will show 
Theorem 1: Suppose $X, X_{1}, \ldots, X_{m}$ are non-negative, i.i.d. random variables with NBU distribution function. Then

$$
P\{\mathrm{X} \in \mathcal{P}(M, f)\} \geq P\{X \geq f(\hat{M})\}
$$

where

$$
\hat{M}=\arg \max \left\{f(A): A \in 2^{M}\right\} .
$$

Proof: Note that if $f$ is monotone, then $f(\hat{M})=f(M)$. Hereafter we will assume that $f(\hat{M})>0$, otherwise the above bound is trivially true (from (7), since $X$ is non-negative and $\mathbf{x}=0$ is feasible). Now construct a sequence of increasing sets $S_{j}, j=1, \ldots, k$ such that $S_{0}=\emptyset$ and

$$
S_{j}=\arg \min \left\{|A|: S_{j-1} \subset A \subseteq \hat{M} ; f(A)>f\left(S_{j-1}\right)\right\}, j=1, \ldots, k,
$$

where $f\left(S_{k}\right)=f(\hat{M})$. Since $f(\hat{M})>0$ (by assumption) we have $k \geq 1$. Set

$$
\mathcal{Q}(\mathbf{S}, f)=\left\{\mathbf{x}: \sum_{i \in\left\{S_{j}-S_{j-1}\right\}} x_{i} \geq f\left(S_{j}\right)-f\left(S_{j-1}\right), j=1, \ldots, k ; \mathbf{x} \in \mathcal{R}_{+}^{m}\right\}
$$

Then (we will soon see that)

$$
\mathcal{Q}(\mathrm{S}, f) \subset \mathcal{P}(M, f)
$$

Therefore

$$
P\{\mathrm{X} \in \mathcal{P}(M, f)\} \geq P\{\mathrm{X} \in \mathcal{Q}(\mathbf{S}, f)\}
$$

Since

$$
\begin{aligned}
P\{\mathbf{X} \in \mathcal{Q}(\mathrm{S}, f)\} & =P\left\{\sum_{i \in\left\{S_{j}-S_{j-1}\right\}} X_{i} \geq f\left(S_{j}\right)-f\left(S_{j-1}\right), j=1, \ldots, k\right\} \\
& \geq P\left\{X_{j} \geq f\left(S_{j}\right)-f\left(S_{j-1}\right), j=1, \ldots, k\right\} \\
& \geq P\left\{X \geq \sum_{j=1}^{k} f\left(S_{j}\right)-f\left(S_{j-1}\right)\right\}
\end{aligned}
$$


(where the last inequality follows from the NBU property), from (14) one sees that

$$
P\{\mathrm{X} \in \mathcal{P}(M, f)\} \geq P\{X \geq f(\hat{M})\}
$$

To establish (13) we need to show that for any $A \in 2^{M}$, and $\mathbf{x} \in \mathcal{Q}(\mathbf{S}, f)$,

$$
\sum_{j \in A} x_{j} \geq f(A)
$$

For any given set $A \in 2^{M}$, define

$$
B_{j}=A \cap S_{j}, j=0, \ldots, k
$$

Since $B_{k} \subseteq S_{k}$, by the supermodularity of $f$ we have

$$
f\left(\left(A-B_{k}\right) \cup S_{k}\right)-f\left(S_{k}\right) \geq f(A)-f\left(B_{k}\right) .
$$

Since $f\left(S_{k}\right) \geq f(\hat{A})$ for any $\hat{A} \in 2^{M}$ we have from (19)

$$
f\left(B_{k}\right) \geq f(A) .
$$

Therefore if $\sum_{j \in B_{k}} x_{j} \geq f\left(B_{k}\right)$, then by the positivity of $\mathbf{x}$, one will have $\sum_{j \in A} x_{j} \geq f(A)$. Next consider

$$
\begin{aligned}
f\left(B_{k}\right) & =\sum_{j=1}^{k} f\left(B_{j}\right)-f\left(B_{j-1}\right) \\
& \leq \sum_{j=1}^{k} f\left(\left(B_{j}-B_{j-1}\right) \cup S_{j-1}\right)-f\left(B_{j-1}\right),
\end{aligned}
$$

where the last inequality follows from the supermodularity of $f$. Observe that $\left(B_{j}-B_{j-1}\right) \cup$ $S_{j-1} \subset S_{j}$ and therefore (from the construction of these sets) one has $f\left(\left(B_{j}-B_{j-1}\right) \cup\right.$ $\left.S_{j-1}\right)-f\left(B_{j-1}\right) \leq 0$ if $\left(B_{j}-B_{j-1}\right) \cup S_{j-1}$ is strictly a subset of $S_{j}$ and otherwise (from the definition of $\mathcal{Q}(\mathrm{S}, f)$ ) one has $f\left(S_{j}\right)-f\left(S_{j-1}\right) \leq \sum_{i \in\left\{S_{j}-S_{j-1}\right\}} x_{i}=\sum_{i \in B_{j}-B_{j-1}} x_{i}$. Then from (21) and the non-negativity of $\mathbf{x}$ one has

$$
\begin{aligned}
f\left(B_{k}\right) & \leq \sum_{j=1}^{k} f\left(\left(B_{j}-B_{j-1}\right) \cup S_{j-1}\right)-f\left(B_{j-1}\right) \\
& \leq \sum_{j=1}^{k} \sum_{i \in\left\{B_{j}-B_{j-1}\right\}} x_{i}=\sum_{i \in B_{k}} x_{i} .
\end{aligned}
$$


Therefore $\sum_{i \in A} x_{i} \geq \sum_{i \in B_{k}} x_{i} \geq f\left(B_{k}\right) \geq f(A) . \|$

\section{Application to Transportation Problems}

In this section we will apply the bound developed in Section 2 to transportation problems with random supply and/or demand. Suppose we have a set $K=\{1, \ldots, k\}$ of $k$ supply nodes and a set $N=\{1, \ldots, n\}$ of $n$ demand nodes. Let $r_{i j}$ be the indicator that node $i$ can supply the demand node $j\left(i \in K\right.$ and $j \in N$ ). (i.e., $r_{i j}$ takes the value 1 if the supply is possible and 0 otherwise.)

Suppose the demand at node $j$ is $d_{j}, j \in N$ and the supply available at the supply node $i$ is $s_{i}, i \in K$. Then the total supply available to the demand nodes in $A \subseteq N$ is given by

$$
s(A)=\sum_{i \in N} s_{i} r_{i}(A)
$$

where

$$
r_{i}(A)=\left[1-\Pi_{j \in A}\left(1-r_{i j}\right)\right],
$$

takes the value 1 if supply node $i$ can supply at least one demand node in $A$ and zero otherwise. Since $r: 2^{N} \rightarrow\{0,1\}$ is a submodular set function, it is easily seen that one has

Lemma 1: The set function $s: 2^{N} \rightarrow \mathcal{R}_{+}$is submodular.

A given supply $\mathbf{s}=\left(s_{1}, \ldots, s_{k}\right)$ is feasible (i.e., there exists a feasible solution to the associated sparse transportation problem) iff

$$
s(A) \geq d(A), A \subseteq N,
$$

where

$$
d(A)=\sum_{j \in A} d_{j}, A \subseteq N
$$

This condition is essentially a restatement of Hall's Theorem (see Hall 1935). 
FIXED SUPPLY NODES (K):

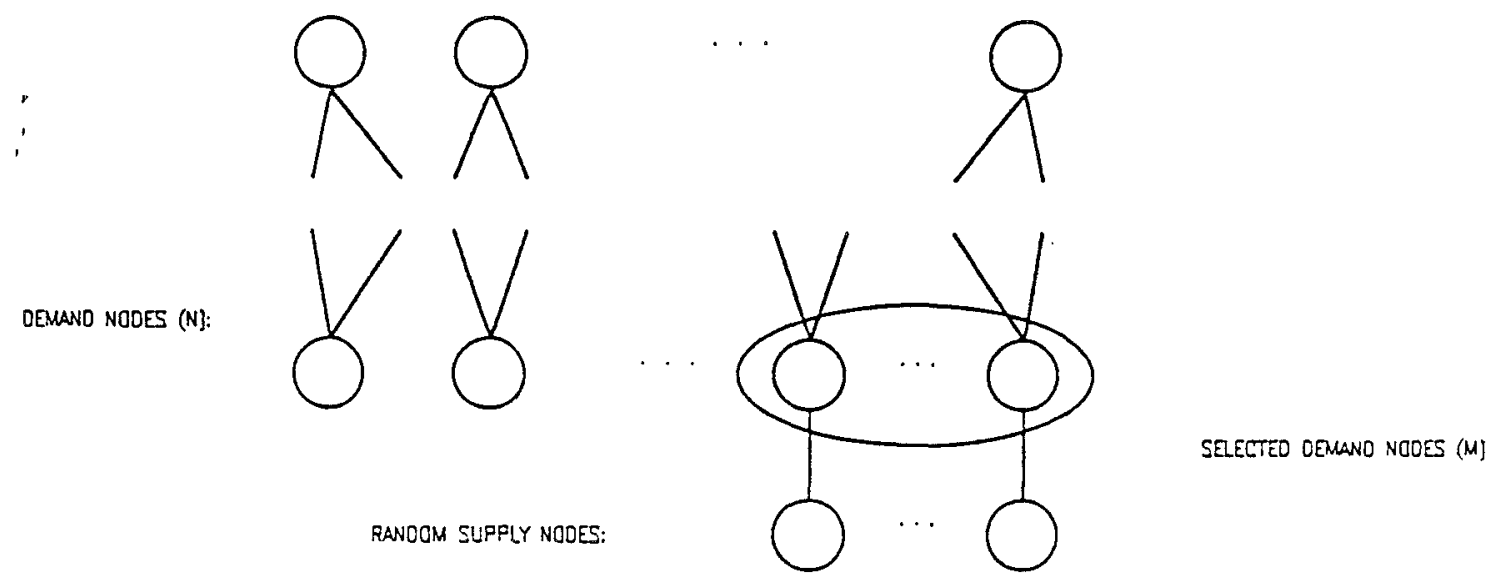

Figure 3: Transportation Network with Outside Sources

First we will consider a situation, illustrated in Figure 3, where we have a fixed supply of $s_{\boldsymbol{i}}$ units available at the supply node $i, i \in K$ and a random supply of $X_{j}$ units is available to the demand node $j, j \in M$ for a fixed $M \subseteq N$ from sources outside of the $k$ supply nodes (say from nodes $k+j, j \in M$ such that $r_{(k+j), j}=1, j \in M$ and 0 otherwise). The probability that this supply is feasible is then

$$
\alpha P\{\mathrm{X} \in \mathcal{P}(M, f)\}
$$

where

$$
f(A)=\max \{d(A \cup B)-s(A \cup B): B \subseteq N-M\}, A \subseteq M,
$$

and

$$
\alpha=I\{s(A) \geq d(A), A \subseteq N-M\}
$$


Now suppose we have only one outside supply of a random quantity $X$ that can be supplied (say from node $M$ ) to any of the demand nodes in $M$ (i.e., $r_{M, j}=1, j \in M$ and zero otherwise). Then the probability that this supply is feasible is

$$
\alpha P\{X \geq f(\hat{M})\}
$$

where

$$
\hat{M}=\arg \max \{f(A): A \subseteq M\}
$$

We will next compare the above two cases.

Lemma 2: Suppose $X, X_{1}, \ldots, X_{m}$, are non-negative, i.i.d. random variables with NBU distribution. Then with a fixed supply of $\mathbf{s}$ the probability of supplying the demand with a random supply of $X_{j}$ units to node $j, j \in M$ from other sources is greater than or equal to the probability of supplying the demand with a random supply of $X$ units to the nodes in $M$.

Proof: The result will follow from Theorem 2, (27) and (31) by showing that $f(A)$ defined by (29) is supermodular. To prove supermodularity, we first note that given a supermodular [submodular] function $g: 2^{N} \rightarrow \mathcal{R}$ and an $M \subseteq N$, we have $\hat{g}: 2^{M} \times 2^{N-M} \rightarrow \mathcal{R}$ defined by $\hat{g}(A, B)=f(A \cup B), A \subseteq M ; B \subseteq N-M$ is supermodular [submodular]. Theorem 4.3 of Topkis (1978) implies that given such a $\hat{g}(A, B), g^{\prime}(A)=\operatorname{Max}\{g(A, B): B \subseteq N-M\}$ is also supermodular [submodular]. Now from (26) it is clear that $d(A \cup B)$ is supermodular and from Lemma 1 it is clear that $s(A \cup B)$ is submodular in $A \subseteq M ; B \subseteq N-M$. Therefore, the Max operation in (29) gives a supermodular function. \|

We are now ready to present the main result of this section.

Theorem 2: Suppose we have a fixed demand of $d_{j}$ units at node $j, j \in N$ and have a random supply of $S_{i}$ units at node $i, i \in K$. Let $\gamma$ be the probability that this random supply is feasible. If $S_{1}, \ldots, S_{k}$, are independent and have NBU distribution, then

$$
\gamma \leq \Pi_{j=1}^{n} P\left\{Z_{j} \geq d_{j}\right\}
$$


where

$$
Z_{j}=\sum_{i \in K} S_{i} r_{i j}, j \in N
$$

Pro,of: Suppose node $i$ can supply all and only the nodes in $M \subseteq N$ (i.e., $r_{i j}=1, j \in M$ and zero otherwise). Then

$$
P\left\{\text { Supply is feasible } \mid S_{l}=s_{l}, l=1, \ldots, k ; l \neq i\right\}=\alpha P\left\{S_{i} \geq f(\hat{M})\right\}
$$

where $\alpha, f$ and $\hat{M}$ are as defined above with $s_{i}=0$. Then from Lemma 2 one sees that

$$
P\left\{\text { Supply is feasible } \mid S_{l}=s_{l}, l=1, \ldots, k ; l \neq i\right\} \leq
$$

$P\left\{\right.$ Supply is feasible $\mid S_{l}=s_{l}, l=1, \ldots, k ; l \neq i$ and the demand is $\left.d_{j}-S_{i}^{(j)}, j \in M\right\}$, where $S_{i}, S_{i}^{(1)}, \ldots, S_{i}^{(m)}$, are i.i.d. random variables. Now unconditioning (36) with respect to $s$ one gets,

$$
\gamma \leq P\left\{\text { Supply is feasible } \mid S_{i}=0 \text { and the demand is } d_{j}-S_{i}^{(j)}, j \in M\right\}
$$

Continuing this for all other supply nodes one gets

(38) $\gamma \leq P\left\{\right.$ Supply is feasible $\mid S_{i}=0, i \in K$ and the demand is $\left.d_{j}-\sum_{i \in K} S_{i}^{(j)} r_{i j}, j \in N\right\}$.

Since $P\left\{\right.$ Supply is feasible $\mid S_{i}=0, i \in K$ and the demand is $\left.d_{j}-\sum_{i \in K} S_{i}^{(j)} r_{i j}, j \in N\right\}$ $=\Pi_{j=1}^{n} P\left\{Z_{j} \geq d_{j}\right\}$, the proof is complete. $\|$

Finally, we note that this result can be extended to the case of random supplies.

Corollary 1: Theorem 2 is true even when the demands are given by mutually independent random variables $D_{j}$ for $j \in N$.

Proof: Conditioning $D_{j}=d_{j}, j \in N$ the proof follows immediately from Theorem $2 . \|$ 

